\title{
NUMERICAL METHOD FOR DARCY FLOW DERIVED USING DISCRETE EXTERIOR CALCULUS
}

\author{
ANIL N. HIRANI, KALYANA B. NAKSHATRALA, AND JEHANZEB H. CHAUDHRY
}

\begin{abstract}
We derive a numerical method for Darcy flow, hence also for Poisson's equation in first order form, based on discrete exterior calculus (DEC). Exterior calculus is a generalization of vector calculus to smooth manifolds and DEC is its discretization on simplicial complexes such as triangle and tetrahedral meshes. We start by rewriting the governing equations of Darcy flow using the language of exterior calculus. This yields a formulation in terms of flux differential form and pressure. The numerical method is then derived by using the framework provided by DEC for discretizing differential forms and operators that act on forms. We also develop a discretization for spatially dependent Hodge star that varies with the permeability of the medium. This also allows us to address discontinuous permeability. The matrix representation for our discrete non-homogeneous Hodge star is diagonal, with positive diagonal entries. The resulting linear system of equations for flux and pressure are saddle type, with a diagonal matrix as the top left block. Our method requires the use of meshes in which each simplex contains its circumcenter. The performance of the proposed numerical method is illustrated on many standard test problems. These include patch tests in two and three dimensions, comparison with analytically known solution in two dimensions, layered medium with alternating permeability values, and a test with a change in permeability along the flow direction. A short introduction to the relevant parts of smooth and discrete exterior calculus is included in this paper. We also include a discussion of the boundary condition in terms of exterior calculus.
\end{abstract}

\section{INTRODUCTION}

We have discretized the equations of Darcy flow and obtained a numerical method on staggered mesh pairs with fluxes and pressures being the primary variables. The numerical method was obtained by using discrete exterior calculus (DEC) [22, 23, 28]. Exterior calculus generalizes vector calculus to higher dimensions and to smooth manifolds [1] and DEC is its discretization. This discretization yields numerical methods for solving partial differential equations (PDEs) on simplicial complexes, such as triangle,

Date: October 18, 2008, Version 1.5264.

2000 Mathematics Subject Classification. Primary 65N30, 76S05; Secondary 53-04, 5504 .

Key words and phrases. discrete exterior calculus; mixed method; finite element method; finite volume method; Darcy flow; Poisson's equation. 
tetrahedral or higher dimensional simplicial meshes. A recent implementation of DEC is described in [7]. DEC is related to many discretizations of exterior calculus that have been popular in or are currently being pursued in numerical analysis. Others include finite element exterior calculus [4], mimetic and compatible discretizations of PDEs [10, 30], and the covolume method [40]. See [3] for a collection of recent papers in these fields. Those parts of exterior calculus and DEC that are relevant to this paper are summarized in Section 2.

The equations of Darcy flow model the flow of a viscous incompressible fluid in a porous medium. The equations consist of Darcy's law (which expresses force balance), the continuity equation, and the boundary condition. The first two form a very simple pair of equations, being Poisson's equation in first order form. In this case Darcy flow can be rewritten as Poisson's equation with pressure as the unknown. In applications however, it is often the velocity field or flux that is of primary interest and there is a loss of smoothness and accuracy in going from pressure to velocity. In first order form (i.e., when Darcy law and the continuity equations are not combined into a single equation) Darcy flow equations can be discretized using mixed finite element or volume methods.

In mixed methods, velocity (or flux) along with pressure are taken to be the primary variables and this can yield more accurate results compared to the pressure-only formulation. Mixed formulations require careful choice of spaces for velocity and pressure since not all combinations yield stable methods. For example, the use of continuous piecewise linear representation for both velocity and pressure results in an unstable method [16, 18]. An example of such unstable behavior is shown in Figure 1. Many fixes for such instability are presented in the literature. For example, one can use RaviartThomas (RT) elements [43], Nédélec elements [39], Brezzi-Douglas-Marini (BDM) elements [17], or a variety of other finite element spaces summarized in [19]. Many of these spaces that have yielded stable discretizations of Darcy flow and other problems have been unified under the umbrella of finite element exterior calculus [4]. Stabilized mixed finite elements methods have also been developed for Darcy flow [9, 29, 35, 37, 38]. Finite volume [2] and covolume methods [20, 21, 40] are yet another approach for solving the equations of Darcy flow. A monotone, locally conservative finite volume scheme for diffusion equation is described in [33] and a mimetic finite difference scheme is in [32].

The primary variables in our method are area or volume flux (depending on whether the problem is $2 \mathrm{D}$ or $3 \mathrm{D}$ ), and pressure. The fluxes are placed on edges in triangle meshes or triangles in tetrahedral meshes. This leads to pressures being placed at circumcenters of top dimensional simplices. Thus the pressure can be considered constant in each simplex. Our method is related to the methods for diffusion described in [42] and to the covolume method applied to Darcy flow in $[20,21]$ but our emphasis is on the relationships between the numerical method and exterior calculus. In addition 


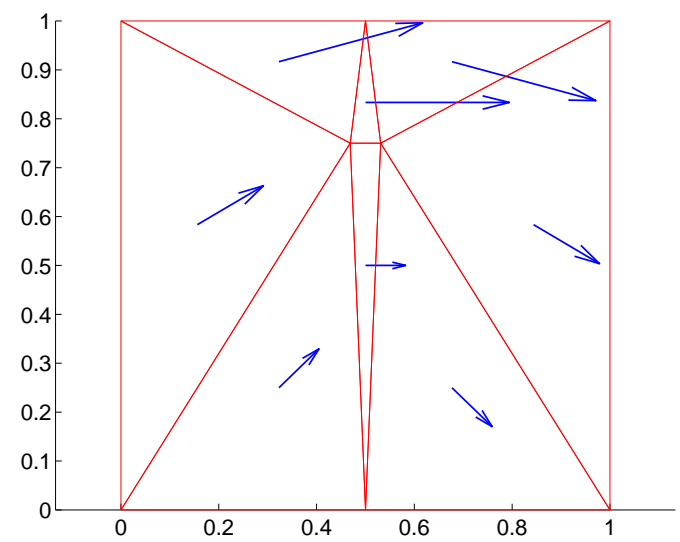

Figure 1: Mixed finite element method can be used to solve Darcy flow and it is well-known that care is needed in selecting the underlying finite element spaces. For example, equal order interpolation for both velocity and pressure is unstable. Here we show results for such a choice using piecewise linear finite elements. The correct solution is the constant vector field $(1,0)$. The fix for such unstable behavior is well-known in the finite elements literature $[16,18]$. In this paper we provide a related but different numerical method based on discrete exterior calculus.

we treat discontinuous permeability explicitly. The treatment of flux and pressure in our method is similar to that for Navier-Stokes equations in [25], which is also based on DEC. The presentation in this paper depends on well-centered meshes $[44,45,46]$ although a weaker requirement on meshes may be sufficient as in covolume methods [40, 41].

Our results: Our discretization space is similar to the lowest order RaviartThomas elements. However, the linear system matrix resulting from our discretization is a saddle type matrix [8] with a diagonal matrix as the top left block. Our method enforces mass balance locally and globally and passes several standard numerical tests. In $2 \mathrm{D}$ we show that the method passes patch test involving constant velocity and linear pressure. We also compare our numerical solution with an analytical solution for a more general source term. We develop a diagonal discretization of spatially dependent Hodge star that depends on the permeability. This is used for the case of layered medium with alternating permeabilities, and for a discontinuous medium where we use different pairs of permeability under constant velocity conditions. We also show that our method passes patch tests even in the 3D case. These numerical results are in Section 6 and the advantages of a DEC based approach are discussed in Section 7. A discussion of boundary conditions in terms of exterior calculus is in Sections 3 and 6.1. 


\section{Review of Discrete Exterior Calculus (DEC)}

In this section we briefly outline the relevant parts of smooth and discrete exterior calculus. We discuss only the operators that are relevant for Darcy flow. For more details on DEC see [22, 23, 28] and for details on exterior calculus see $[1,5]$. In Sections 4 and 5 we describe our method for solving equations of Darcy flow so that it can be implemented without knowledge of DEC or exterior calculus. Thus, a reader unfamiliar with some of the terms used in this section should still be able to follow and implement the method. One useful characteristic of exterior calculus is that all objects and operators can be expressed in coordinate independent fashion. This aspect however is harder to explain in a few paragraphs. Instead, we give some examples using coordinates to describe the operators and objects of exterior calculus.

2.1. Smooth exterior calculus. As mentioned earlier, exterior calculus generalizes vector calculus to smooth manifolds $[1,5]$ and it consists of operators on smooth general tensor fields defined on manifolds. A tensor field evaluated at a point is a multilinear function on the tangent space, mapping vector and covector arguments to $\mathbb{R}$ (the set of real numbers). Other ranges besides $\mathbb{R}$ are possible but not relevant here. Vector fields, symmetric tensor fields such as metrics and antisymmetric tensor fields are all examples of tensors. Antisymmetric tensors have been singled out in exterior calculus and are called differential forms. A differential $k$-form when evaluated at a point is an antisymmetric multilinear map on the tangent space that takes $k$ vector arguments and produces a real number. It is an object that can be integrated on a $k$-dimensional space. In exterior calculus, it only makes sense to integrate differential $k$-forms on a $k$-manifold.

Let $M$ be an $n$-dimensional orientable Riemannian manifold (a manifold with an inner product on the tangent space at each point), $T M$ the tangent bundle (disjoint union of the tangent spaces at all points of $M$ ), $\mathfrak{X}(M)$ the space of smooth vector fields and $\Omega^{k}(M)$ the space of differential $k$-forms on $M$.

Then exterior derivative is a map $\mathrm{d}_{k}: \Omega^{k}(M) \rightarrow \Omega^{k+1}(M)$ (sometimes written without the subscript) that raises the degree of a form, and the wedge product is a map or binary operator $\wedge: \Omega^{k}(M) \times \Omega^{l}(M) \rightarrow \Omega^{k+l}(M)$ that combines differential forms. The most important property of $\mathrm{d}$ is that $\mathrm{d}_{k+1} \circ \mathrm{d}_{k}=0$. These two operators are enough to describe a basis for differential forms on $M$. Taking $M=\mathbb{R}^{3}$ (the standard three dimensional Euclidean space), with standard metric and coordinates $x, y$ and $z$, a basis for $\Omega^{1}\left(\mathbb{R}^{3}\right)$, the space of 1 -forms is $(d x, d y, d z)$ and a basis for $\Omega^{2}\left(\mathbb{R}^{3}\right)$ is $(d x \wedge d y, d x \wedge d z, d y \wedge d z)$. Let $f$ be a scalar valued function on $\mathbb{R}^{3}$ (i.e., a 0 -form). Then its exterior derivative $\mathrm{d} f$ equals its differential $d f$ and is

$$
\mathrm{d} f=\frac{\partial f}{\partial x} d x+\frac{\partial f}{\partial y} d y+\frac{\partial f}{\partial z} d z
$$


For a 1-form $\alpha=\alpha_{1} d x+\alpha_{2} d y+\alpha_{3} d z$, where $\alpha_{i}$ are scalar valued functions, its exterior derivative is

$\mathrm{d} \alpha=\left(\frac{\partial \alpha_{2}}{\partial x}-\frac{\partial \alpha_{1}}{\partial y}\right) d x \wedge d y+\left(\frac{\partial \alpha_{3}}{\partial x}-\frac{\partial \alpha_{1}}{\partial z}\right) d x \wedge d z+\left(\frac{\partial \alpha_{3}}{\partial y}-\frac{\partial \alpha_{2}}{\partial z}\right) d y \wedge d z$.

The Hodge star is an isomorphism, $*: \Omega^{k}(M) \rightarrow \Omega^{n-k}(M)$. For $\mathbb{R}^{3}$ with standard metric, the Hodge star satisfies the following properties:

$$
\begin{aligned}
& * 1=d x \wedge d y \wedge d z \\
& * d x=d y \wedge d z, \quad * d y=-d x \wedge d z, \quad * d z=d x \wedge d y \\
& *(d y \wedge d z)=d x, \quad *(d x \wedge d z)=-d y, \quad *(d x \wedge d y)=d z \\
& *(d x \wedge d y \wedge d z)=1 .
\end{aligned}
$$

For $\mathbb{R}^{2}$ the equivalent properties are

$$
\begin{aligned}
& * 1=d x \wedge d y \\
& * d x=d y, \quad * d y=-d x ; \\
& *(d x \wedge d y)=1
\end{aligned}
$$

An important property of Hodge star is that for a $k$-form $\alpha$,

$$
* * \alpha=(-1)^{k(n-k)} \alpha \text {. }
$$

Another operator relevant for Darcy flow is flat, which is a map $b$ : $\mathfrak{X}(M) \rightarrow \Omega^{1}(M)$ that identifies vector fields and 1-forms via the metric. Consider $\mathbb{R}^{3}$ with the standard inner product, and standard orthonormal basis. Then for a vector field $V$ with components $V_{1}, V_{2}$ and $V_{3}$, we have $V^{b}=V_{1} d x+V_{2} d y+V_{3} d z$. If the inner product is not the standard one or the basis is not orthonormal then the relationship between a vector field and its flat in coordinates is more complicated. In $\mathbb{R}^{3}$, for a scalar function $f$ and vector field $V$, some important relationships involving flat operator are:

$$
(\nabla f)^{b}=\mathrm{d} f, \quad(\operatorname{curl} V)^{b}=* \mathrm{~d} V^{b}, \quad \operatorname{div} V=* \mathrm{~d} * V^{b} .
$$

Thus div, grad and curl can be defined in terms of exterior calculus operators. Note that the operators $d$ and $\wedge$ are metric independent and so they can be defined on a manifold without having to define a Riemannian metric. On the other hand, the operators $*$ and $b$ do require a metric for their definition.

2.2. Primal and dual mesh. Discretizing exterior calculus involves deciding what should replace the smooth manifolds, differential forms and other tensor fields and operators that act on these. Recall that a simplicial complex $K$ in $\mathbb{R}^{N}$ is a collection of simplices in $\mathbb{R}^{N}$ such that every face of a simplex of $K$ is in $K$ and such that the intersection of any 2 simplices of $K$ is a face of each of them. The dimension $n$ of the complex is the highest dimension of its simplices. Thus $n \leq N$ where $N$ is the dimension of the embedding space. In DEC, the oriented Riemannian manifolds $M$ of smooth exterior calculus is replaced by an oriented manifold simplicial 
complex $K$ as described in [28]. Briefly, these are simplicial complexes in which the neighborhood of every interior point is homeomorphic to ("looks like") an open subset of $\mathbb{R}^{n}$ and in which each simplex of dimension $k$, for $0 \leq k \leq n-1$, is a face of some $n$-simplex. Thus a triangle with an edge sticking out from one vertex would not be admissible and neither would a triangle mesh surface with a fin like triangle sticking out from an edge. In addition all the $n$-simplices must have the same orientation. See [28] for details. An oriented manifold simplicial complex will be also called a primal mesh.

In addition to the primal mesh $K$ a staggered cell complex $\star K$ associated with $K$ and referred to as the dual mesh also plays a role in DEC. An example of a primal mesh, with some pieces of the dual mesh highlighted is shown in Figure 2. Usually the dual mesh is not explicitly stored. What are needed instead are lengths, areas or volumes of pieces of the dual mesh, the specific needed quantities depending on the application. The primal mesh is a simplicial complex, i.e., a triangle or tetrahedral (or higher dimensional) mesh such as are used in finite volume or finite element methods. One special requirement is that the circumcenter of each simplex be in the interior of the simplex. Such a mesh is called well-centered and in 2D all its triangles are acute angled. While this is restrictive, there now exist two classes of algorithms that can produce such meshes in many cases. One class is represented by [45] in which a planar triangle mesh can be obtained starting from a given mesh, using an optimization approach. In [46], the authors extend the method to higher dimensions and give examples of simple well-centered tetrahedral meshes and in [44] some simple 3D shapes are triangulated using well-centered tetrahedra. Most of the meshes used in this paper are a result of the work in $[44,45,46]$. Another class of algorithms produces a planar well-centered mesh starting with the description of the boundary $[26,34,49]$. The well-centeredness restriction on meshes can be removed, possibly at the cost of acquiring off diagonal terms in the discrete Hodge star [7], or a discrete Hodge star matrix with different conditioning. For Darcy flow in particular, it may be possible to weaken the conditions on the meshes, but we do not pursue these topics in this paper.

In what follows, $\sigma^{k}$ will be a primal $k$-simplex, a $k$-dimensional simplex in the primal mesh. The corresponding dual $(n-k)$-cell, an $(n-k)$-dimensional cell in the dual mesh will be denoted by $\star \sigma^{k}$. We will use $\operatorname{cc}\left(\sigma^{k}\right)$ to mean the circumcenter of $\sigma^{k}$, the unique point equidistant from all vertices of $\sigma^{k}$. The notation $\sigma \prec \tau$ will mean that simplex $\sigma$ is a face of simplex $\tau$ and $\tau \succ \sigma$ will mean that $\tau$ contains $\sigma$ as one of its faces.

To find the dual cell $\star \sigma^{k}$ of the primal simplex $\sigma^{k}$ proceed as follows. Start from the circumcenter $\operatorname{cc}\left(\sigma^{k}\right)$. Traverse in straight lines, one by one, to the circumcenters $\operatorname{cc}\left(\sigma^{k+1}\right)$ of all $\sigma^{k+1} \succ \sigma^{k}$ and from those to the next dimension and so on all the way to the top dimension. Each path of these traversals yields a simplex of dimension $n-k$. The union of all such simplices is the dual cell $\star \sigma^{k}$ of simplex $\sigma^{k}$. For example, in Figure 2, the dual of an 


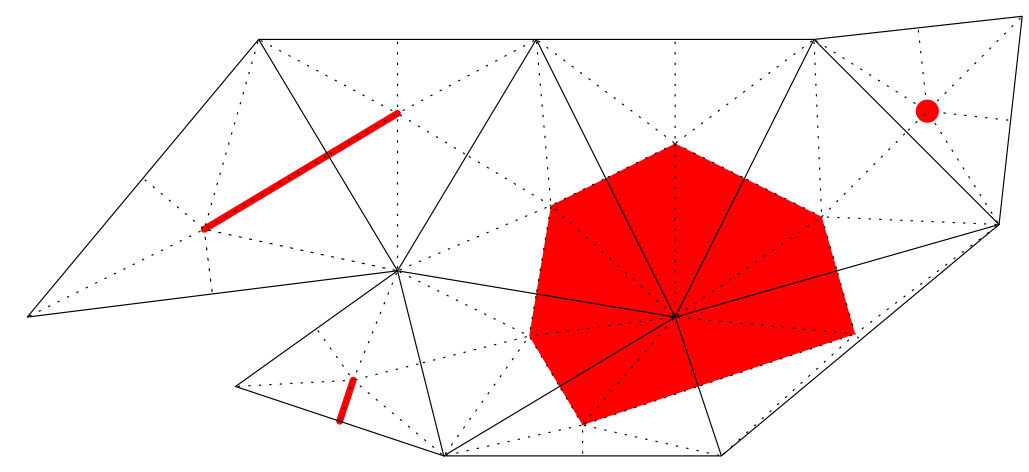

Figure 2: A simplicial complex is subdivided using circumcentric subdivision and the dual cells are constructed from the subdivision. The new edges introduced by the subdivision are shown dotted. The dual cells shown are colored red. Figure taken from [28].

internal edge is obtained by starting from its middle and traversing to the circumcenters of the two triangles containing it. The resulting two edges together form the dual of the edge. Note that it need not be a straight line if the two adjacent triangles do not lie in the same affine space. See [28] for more examples of primal-dual pairs.

The primal and dual meshes of DEC are oriented. The primal mesh is oriented consistently at the top level. For example, either all the triangles in a triangle mesh must be oriented clockwise, or all of them must be oriented counter-clockwise. Similarly, all the tetrahedra in a tetrahedral mesh must be right-handed, or all must be left-handed. The lower dimensional simplices can be oriented arbitrarily, for example, using the dictionary order of the vertex numbers. The orientation of the dual cells is implied by the orientation of the corresponding primal simplices and of the top level primal simplices. For details see [28]. For triangle meshes, a vector along the primal edge followed by one along the dual edge should define the same orientation as that of the triangles. For tetrahedral meshes the orientation of a face followed by a vector along the dual edge should form the same handedness as that of the tetrahedron.

2.3. Chains and boundary operator. Let $K$ be a finite simplicial complex. Recall from algebraic topology [36] that a $k$-chain on $K$ is a function from the set of oriented $k$-simplices of $K$ to the set of integers $\mathbb{Z}$. A $k$-chain $c$ has the property that that $c(\sigma)=-c(-\sigma)$, where $-\sigma$ is $\sigma$ with the opposite orientation. Chains are added by adding their values. The space of $k$-chains is a group and is denoted $C_{k}(K)$. The group structure will not be important to us except for the fact that it will allow us to talk about homomorphisms - maps between groups that preserve the group structure. For a $k$-simplex $\sigma^{k}$ we will use $\sigma^{k}$ or $\sigma$ to denote the simplex as well as the 
chain that takes the value 1 on the simplex and 0 on all other $k$-simplices in $K$. Such a chain is called an elementary $k$-chain.

The boundary operator $\partial_{k}: C_{k}(K) \rightarrow C_{k-1}(K)$ is defined as a homomorphism (that is, $\left.\partial_{k}(a+b)=\partial_{k} a+\partial_{k} b\right)$ by defining it on oriented simplices $\left[v_{0}, \ldots v_{k}\right]$. It is defined by

$$
\partial_{k}\left[v_{0} \ldots v_{k}\right]=\sum_{i=0}^{k}(-1)^{i}\left[v_{0}, \ldots, \widehat{v}_{i}, \ldots, v_{k}\right]
$$

the hat indicating the missing vertex. For example if $\left[v_{0}, v_{1}, v_{2}\right]$ is a triangle in $\mathbb{R}^{2}$, oriented counterclockwise, then the boundary of the corresponding elementary 2 -chain is the sum of the 3 elementary 1-chains $\left[v_{1}, v_{2}\right]$, $-\left[v_{0}, v_{2}\right]\left(=\left[v_{2}, v_{0}\right]\right)$ and $\left[v_{0}, v_{1}\right]$.

2.4. Cochains as discrete forms. As is usual in most discretizations of exterior calculus, in DEC discrete differential $k$-forms are defined to be elements of $\operatorname{Hom}\left(C_{k}(K), \mathbb{R}\right)$. This is the space of real-valued homomorphisms on the space of $k$-chains. This space is called the space of $k$-cochains or discrete differential $k$-forms. Thus given $k$-chains $a$ and $b$ and a $k$-cochain $\alpha$, we have $\alpha(a+b)=\alpha(a)+\alpha(b)$ in which each term is a real number. The space of primal $k$-cochains on a simplicial complex $K$ will be denoted by $C^{k}(K ; \mathbb{R})$ and the dual $k$-cochains on the dual cell complex $\star K$ by $C^{k}(\star K ; \mathbb{R})$. We will shorten these notations to $C^{k}(K)$ and $C_{k}(\star K)$. Discrete forms are created from (piecewise) smooth forms with the de Rham map $R: \Omega^{k}(K) \rightarrow C^{k}(K)$ or $R: \Omega^{k}(K) \rightarrow C^{k}(\star K)$ depending on the context [24]. For a smooth form $\alpha$, we will denote the evaluation of the cochain $R(\alpha)$ on a chain $c$ as $\langle R(\alpha), c\rangle$ and define it as $\int_{c} \alpha$. Thus given a smooth $k$-form $\alpha$ the de Rham map converts it into the $k$-cochain $\int \alpha$ with the slot for integration domain

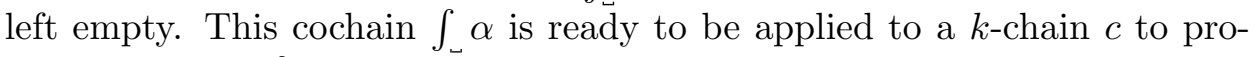
duce a number $\int_{c} \alpha$. Note that we are implicitly assuming that the smooth quantities are defined on the simplicial mesh that is the discretization of the smooth manifold. For planar and spatial domains we consider in the examples in this paper, this condition is trivially true. For more general domains, like surfaces embedded in $\mathbb{R}^{3}$ this restriction can be removed, but work on such generalizations, especially how it affects numerical solutions of PDEs is still in early stages. For some related ideas see [27, 47].

The cochain spaces $C^{k}(K)$ and $C^{k}(\star K)$ defined above are groups, but in addition they are also vector spaces. If there are $N_{k}$ simplices of dimension $k$ in $K$ then the vector space dimension $\operatorname{dim}\left(C^{k}(K)\right)$ is $N_{k}$. Similarly, if there are $N_{k}$ cells of dimension $k$ in $\star K$ then $\operatorname{dim}\left(C^{k}(\star K)\right)$ is $N_{k}$. Note that $\operatorname{dim}\left(C^{k}(K)\right)=\operatorname{dim}\left(C^{n-k}(\star K)\right)$ since $k$-simplices of $K$ are in one-toone correspondence with $(n-k)$-cells of $\star K$. To define a basis for $C^{k}(K)$ as a vector space, the $k$-simplices are first ordered in some way. For example in PyDEC [7], the $k$-simplices are ordered in dictionary order based on the vertex names of the simplex. In a triangle $\left[v_{0}, v_{1}, v_{2}\right]$, the dictionary order 
for edges is $\left[v_{0}, v_{1}\right],\left[v_{0}, v_{2}\right]$ and $\left[v_{1}, v_{2}\right]$. We would typically refer to these edges as $\sigma_{0}^{1}, \sigma_{1}^{1}$ and $\sigma_{2}^{1}$, respectively. If $\sigma_{0}, \ldots, \sigma_{N_{k}}$ is an ordered list of the $k$-simplices of $K$ then we can define a basis $\left(\sigma_{0}^{*}, \ldots, \sigma_{N_{k}}^{*}\right)$ for $C^{k}(K)$ where $\left\langle\sigma_{i}^{*}, \sigma_{j}\right\rangle=\delta_{i j}$, the Kronecker delta. That is, $\sigma_{i}^{*}$ is the $k$-cochain that is 1 on the elementary $k$-chain of $\sigma_{i}$ and 0 on the other elementary $k$-chains in $K$. In computations we represent elements of $C^{k}(K)$ and $C^{k}(\star K)$ as vectors of appropriate dimensions in these bases.

2.5. Discrete exterior derivative and Hodge star. We now give definitions of discrete exterior derivative and discrete Hodge star. These are given here without explanation as to why these are good choices for the discrete operators. Such an explanation can be found in $[22,28]$. The discrete exterior derivative on the primal cochains will also be denoted as $\mathrm{d}$ ( or $\mathrm{d}_{k}$ if the degree of the source space is to be specified) and is defined using the boundary operator in such a way that Stokes theorem is true by definition. For the exterior derivative on the dual cochains we will use the notation $\mathrm{d}^{*}$ $\left(\right.$ or $\left.\mathrm{d}_{k}^{*}\right)$. For a $k$-cochain $\alpha^{k}$ and $(p+1)$-chain $c^{k+1}$, define

$$
\left\langle\mathrm{d}_{k} \alpha^{k}, c^{k+1}\right\rangle:=\left\langle\alpha^{k}, \partial_{k+1} c^{k+1}\right\rangle \text {. }
$$

In the basis for $C^{k}(K)$ described in Section 2.4, the matrix representation of $\mathrm{d}_{k}$, denoted $D_{k}$, is an $N_{k+1}$ by $N_{k}$ matrix with entries 0,1 or -1 . Analogous bases for $C^{k}(\star K)$ using the dual cells yield matrix form of the dual discrete exterior derivative operator. The matrices of the primal and dual exterior derivative are related. In fact the matrix form of $\mathrm{d}_{k}^{*}$ is $\pm D_{n-k-1}^{T}$ where the sign depends on $n$ and $k$. For Darcy flow the only relevant pairs of primal and dual exterior derivatives are $\mathrm{d}_{0}^{*}$ and $\mathrm{d}_{n-1}$ and the matrix form for $\mathrm{d}_{0}^{*}$ is $(-1)^{n} D_{n-1}^{T}$.

The discrete Hodge star can be thought of as an operator that "transfers information" between the primal and dual meshes. Given a $k$-simplex $\sigma$ and a primal $k$-cochain $\alpha$, the discrete Hodge star of $\alpha$ (denoted $* \alpha$ ) is a dual discrete $(n-k)$-cochain defined by its value on the dual cell $\star \sigma$ by

$$
\frac{1}{|\star \sigma|}\langle * \alpha, \star \sigma\rangle:=\frac{1}{|\sigma|}\langle\alpha, \sigma\rangle .
$$

Here $|\sigma|$ is the measure of $\sigma$ and $|\star \sigma|$ is the measure of the circumcentric dual cell corresponding to $\sigma$, with measure of a 0 -dimensional object being 1 . See $[22,28]$ for details. We will sometimes use $*_{k}$ to denote the Hodge star with $C^{k}(K)$ as its domain. Using the bases for $C^{k}(K)$ and $C^{n-k}(\star K)$ mentioned above, the matrix $M_{k}$ for $*_{k}$ is a diagonal $N_{k}$ by $N_{k}$ matrix and for a wellcentered mesh the diagonal entries are all positive. It is helpful to use $*_{k}^{-1}$ to denote the inverse map although the inverse notation is usually not used for smooth Hodge star. To simulate the Hodge star property (2.1) on the discrete side we will always use

$$
(-1)^{k(n-k)} *_{k}^{-1}
$$

whenever the inverse discrete Hodge star map is used. 
The various primal and dual cochain complexes in 2 dimensions are related via the discrete exterior derivative and discrete Hodge star operators in the manner shown in the following diagram:

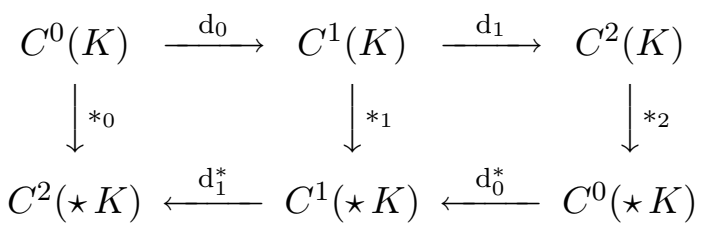

The corresponding diagram for 3 dimensions is:

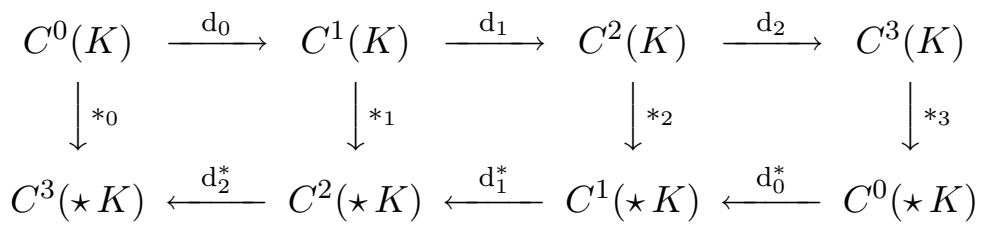

2.6. Interpolation of cochains. To go from cochains to piecewise smooth forms on a simplicial complex $K$ a well-known map is the Whitney map $[11,13,48]$ denoted $W$. This map can be thought of as a way to interpolate numbers defined on edges, triangles and tetrahedra. Thus it goes in direction opposite of the one for the de Rham map which is a discretization map. For a complex $K$ consisting of a triangle $\left[v_{0}, v_{1}, v_{2}\right]$ and its faces, the Whitney map for 1-cochains is defined by extending by linearity, the following to all of $C^{1}(K)$

$$
W\left(\left[v_{i}, v_{j}\right]^{*}\right):=\mu_{i} \mathrm{~d} \mu_{j}-\mu_{j} \mathrm{~d} \mu_{i},
$$

for $i \neq j$ and $i, j \in\{0,1,2\}$. Here $\mu_{i}$ is the barycentric basis function corresponding to $v_{i}$, i.e. the affine function that is 1 on $v_{i}$ and 0 at other vertices. Recall that the 1 -cochain $\left[v_{i}, v_{j}\right]^{*}$ is 1 on the elementary 1-chain of the edge $\left[v_{i}, v_{j}\right]$ and 0 at all other elementary 1-chains. For a tetrahedron $\left[v_{0}, v_{1}, v_{2}, v_{3}\right]$ there are Whitney maps as above for interpolating the edge values and in addition there are Whitney maps for interpolating the triangle values, and these are defined by extension from

$$
W\left(\left[v_{i}, v_{j}, v_{k}\right]^{*}\right):=2\left(\mu_{i} \mathrm{~d} \mu_{j} \wedge \mathrm{d} \mu_{k}-\mu_{j} \mathrm{~d} \mu_{i} \wedge \mathrm{d} \mu_{k}+\mu_{k} \mathrm{~d} \mu_{i} \wedge \mathrm{d} \mu_{j}\right) .
$$

The piecewise smooth forms (smooth in each simplex) constructed using the Whitney map are also known as a Whitney forms. Whitney forms can be used to build a low order finite element exterior calculus $[12,13]$ and in computational electromagnetism the Whitney 1-form and 2-form are also known as edge and face elements respectively [14]. Finite element exterior calculus has now been generalized to general polynomial differential forms [4]. We use the Whitney maps only for interpolating the differential forms so we can plot the corresponding vector field for visualization. The vector field corresponding to the Whitney 1-form in equation (2.8) is obtained by applying a sharp operator to get

$$
\mu_{i} \nabla \mu_{j}-\mu_{j} \nabla \mu_{i}
$$


The vector field corresponding to the Whitey 2-form in equation (2.9) is

$$
2\left(\mu_{i} \nabla \mu_{j} \times \nabla \mu_{k}-\mu_{j} \nabla \mu_{i} \times \nabla \mu_{k}+\mu_{k} \nabla \mu_{i} \times \nabla \mu_{j}\right) .
$$

\section{Governing Equations}

We first present the governing equations of Darcy flow in the standard vector calculus notation, and then rewrite them using differential forms and vector fields (that is, in exterior calculus notation). This latter form is then discretized on a simplicial complex and its dual, which yields a numerical method for Darcy flow.

Let $\Omega \subset \mathbb{R}^{n}$ be a bounded open domain, $\bar{\Omega}$ its closure and $\partial \Omega:=\bar{\Omega} \backslash \Omega$ its boundary, which is assumed to be piecewise smooth. In this paper $n$ (which represents spatial dimensions) can be 2 or 3 . Let $v: \Omega \rightarrow \mathbb{R}^{n}$ be the Darcy velocity [35] (units $\mathrm{m}^{2} /(\mathrm{m} \mathrm{s})=\mathrm{m} / \mathrm{s}$ for $n=2$ or $\mathrm{m}^{3} /\left(\mathrm{m}^{2} \mathrm{~s}\right)=\mathrm{m} / \mathrm{s}$ for $n=3$ ) and let $p: \Omega \rightarrow \mathbb{R}$ be the pressure. The governing equations of Darcy flow can be written as

$$
\begin{aligned}
v+\frac{k}{\mu} \nabla p & =\frac{k}{\mu} \rho g & & \text { in } \Omega, \\
\operatorname{div} v & =\phi & & \text { in } \Omega, \\
v \cdot \hat{n} & =\psi & & \text { on } \partial \Omega,
\end{aligned}
$$

where $k>0$ is the coefficient of permeability of the medium (units $\mathrm{m}^{2}$ for $n=3), \mu>0$ is the coefficient of (dynamic) viscosity of the fluid (units $\mathrm{kg} /(\mathrm{ms})), \rho>0$ is the density of the fluid, $g$ is the acceleration due to externally applied body force (i.e., $\rho g$ is the body force density), $\phi: \Omega \rightarrow \mathbb{R}$ is the prescribed divergence of velocity, $\psi: \partial \Omega \rightarrow \mathbb{R}$ is the prescribed normal component of the velocity across the boundary, and $\hat{n}$ is the unit outward normal vector to $\partial \Omega$. For consistency $\int_{\Omega} \phi d \Omega=\int_{\partial \Omega} \psi d \Gamma$.

Equation (3.1) is Darcy's law, equation (3.2) is the continuity equation and equation (3.3) is the boundary condition. In the above equations, permeability $k$ is assumed to be a scalar constant. In Section 5 we will relax this constraint and allow $k$ to be a scalar valued function of space. The further generalization needed for modeling anisotropic permeability requires $k$ to be a tensor, which is not addressed in this paper. To simplify the treatment, in the rest of this paper we will assume that there is no external force acting on the system, i.e., $g=0$.

The first step in the DEC formulation is to rewrite the governing equations (3.1) - (3.3) in exterior calculus notation. As above, we first assume that the permeability $k$ is a scalar constant. We first apply the flat operator to both sides of equation (3.1), use equation (2.2) for divergence, and then apply Hodge star to both sides of equation (3.2) to obtain (assuming $g=0$ )

$$
\begin{aligned}
v^{b}+\frac{k}{\mu} \mathrm{d}_{0} p=0 & \text { in } \Omega, \\
\mathrm{d}_{n-1}\left(* v^{b}\right)=\phi \omega & \text { in } \Omega,
\end{aligned}
$$




$$
* v^{b}=\psi \gamma \quad \text { on } \partial \Omega
$$

Here $\omega=* 1$ is a volume $n$-form in $\Omega$ and $\gamma$ is the volume $(n-1)$-form on the boundary $\partial \Omega$ and it is defined by requiring

$$
\gamma\left(X_{1}, \ldots, X_{n-1}\right)=\omega\left(\hat{n}, X_{1}, \ldots, X_{n-1}\right),
$$

for all vector fields $X_{1}, \ldots, X_{n-1}$ on the boundary $\partial \Omega$. Note that in going from equation (3.3) to (3.6) we have used the fact that $(v \cdot \hat{n}) \gamma=* v^{b}$. For an explanation of why this is true see [1, page 506]. The definition (3.7) of $\gamma$ has implications on how the orientations affect the sign of the quantity $\psi \gamma$ and this is explained using a concrete example in Section 6. The other quantities in the equations above are as in (3.1) - (3.3). Note that $\phi$ and $\psi$ must satisfy

$$
\int_{\Omega} \phi \omega=\int_{\partial \Omega} \psi \gamma
$$

by Stokes' theorem, which is analogous to the consistency condition stated earlier.

Next we define a differential form which is the volumetric (or volume) flux in $3 \mathrm{D}\left(\right.$ units $\left.\mathrm{m}^{3} /\left(\mathrm{m}^{2} \mathrm{~s}\right)=\mathrm{m} / \mathrm{s}\right)$ or area flux in $2 \mathrm{D}$ (units $\left.\mathrm{m}^{2} /(\mathrm{ms})=\mathrm{m} / \mathrm{s}\right)$. This quantity will be denoted as $f$, which is an $(n-1)$-form, defined by

$$
f:=*\left(v^{b}\right) .
$$

This is appropriate because as mentioned above, $(v \cdot \hat{n}) \gamma=* v^{b}$. Applying Hodge star to both sides of equation (3.4) and replacing $* v^{b}$ by $f$ everywhere we get the governing equations in terms of the flux and pressure, which can be written as

$$
\begin{aligned}
f+\frac{k}{\mu}\left(* \mathrm{~d}_{0} p\right) & =0 & & \text { in } \Omega, \\
\mathrm{d}_{n-1} f & =\phi \omega & & \text { in } \Omega, \\
f & =\psi \gamma & & \text { on } \partial \Omega .
\end{aligned}
$$

Given $k, \mu, \phi, \psi$ and the boundary condition (3.10), the problem statement is to solve equations (3.8) and (3.9) for the flux $f$ and pressure $p$. Equations (3.8)-(3.10) are the ones that we will discretize first in Section 4 using the discrete operators defined in Section 2.4. An equivalent form for equation (3.8) obtained by applying Hodge star to both sides of (3.8) is

$$
* f+(-1)^{n-1} \frac{k}{\mu} \mathrm{d}_{0} p=0 \quad \text { in } \Omega,
$$

and we will also discretize this equation to get an alternative formulation. Here the $(-1)^{n-1}$ sign has come from the double application of Hodge star using equation (2.1). 


\section{Discretization of Equations}

Let $K$ be a simplicial complex that approximates $\Omega$ and $\star K$ the circumcentric dual of $K$ as defined in Section 2.2. Let $L$ be the approximation of the boundary $\partial \Omega$ so that $L$ consists of the $(n-1)$-dimensional boundary faces of $K$. The differential forms $f, k, \phi \omega$ and $\psi \gamma$ in equations (3.8)- (3.10) are discretized as cochains and the operators $d$ and $*$ are replaced by their discrete counterparts described in Section 2.5.

An important point to note when discretizing is the appropriate placement of the cochains. In particular we will place the discrete flux $f$ on the $(n-$ 1)-dimensional primal simplices - edges in triangle mesh and triangles in tetrahedral mesh. Thus $f \in C^{n-1}(K)$. From equation (3.8) this implies that the discrete version of $* \mathrm{~d}_{0} p$ must also be placed on these primal simplices since $k / \mu$ is a scalar here. Thus $* \mathrm{~d}_{0} p \in C^{n-1}(K)$ from which it follows that $\mathrm{d}_{0} p$ is a dual cochain and $\mathrm{d}_{0} p \in C^{n-(n-1)}(\star K)$, that is $\mathrm{d}_{0} p \in C^{1}(\star K)$ is a dual 1-cochain placed on the dual edges. This finally leads to the conclusion that discrete pressure is a dual 0 -cochain, that is, $p \in C^{0}(\star K)$ and thus the pressure must be placed at the circumcenters of the top dimensional simplices.

Since $k$ is a dual 0-cochain we must use the discrete operator $\mathrm{d}_{0}^{*}$ to replace the exterior derivative in equation (3.8). Referring to the diagrams (2.6) and (2.7) it is clear that the discrete Hodge that should be used to replace $*$ in equation (3.8) is $(-1)^{n-1} *_{n-1}^{-1}$, the $(-1)^{n-1}$ sign coming from expression (2.5). Finally, since $f \in C^{n-1}(K)$ clearly the discrete exterior derivative $\mathrm{d}_{n-1}$ will replace the smooth $\mathrm{d}_{n-1}$ when discretizing equation (3.9).

Thus the discretized equations corresponding to equations (3.8)-(3.10) are the very similar looking

$$
\begin{aligned}
f+\frac{k}{\mu}\left((-1)^{n-1} *_{n-1}^{-1} \mathrm{~d}_{0}^{*} p\right) & =0 & & \text { in } K, \\
\mathrm{~d}_{n-1} f & =\phi \omega & & \text { in } K, \\
f & =\psi \gamma & & \text { on } L,
\end{aligned}
$$

where the unknowns and data are the cochains $f \in C^{n-1}(K), p \in C^{0}(\star K)$, $\phi \omega \in C^{n}(K)$ and $\psi \gamma \in C^{n-1}(K)$ where the last one is carried by $L$. The matrix representation of equations (4.1) and (4.2) adjusted for the boundary condition (4.3) is the linear system to be solved which is described next.

Let $f$ be the vector representing the cochain $f$ in the basis

$$
\left(\sigma_{0}^{*}, \ldots, \sigma_{N_{n-1}}^{*}\right)
$$

for $C^{n-1}(K)$ described in Section 2.4. Recall that $\sigma_{i}^{*}$ is the $(n-1)$-cochain that is 0 on $\sigma_{i}$, the $(n-1)$ dimensional simplex number $i$. As mentioned in Section 2.4 the simplices are ordered, for example, in dictionary order [7]. Similarly, let $k, \phi \omega$ and $\psi \gamma$ be the vectors corresponding to the other quantities appearing in equations (4.1)-(4.3). 
To obtain the linear system to solve we first write equations (4.1) and (4.2) in block matrix form using the matrix form of the operators and objects. This yields

$$
\left[\begin{array}{cc}
I & (k / \mu)(-1)^{(n-1)} M_{n-1}^{-1}\left[\mathrm{~d}_{0}^{*}\right] \\
D_{n-1} & 0
\end{array}\right]\left[\begin{array}{l}
f \\
p
\end{array}\right]=\left[\begin{array}{c}
0 \\
\phi \omega
\end{array}\right],
$$

where $I$ is an $N_{n-1} \times N_{n-1}$ identity matrix, 0 is an $N_{n} \times N_{n}$ zero matrix and $\left[\mathrm{d}_{0}^{*}\right]$ is the matrix form of $d_{0}$. Then using the fact that $\left[\mathrm{d}_{0}^{*}\right]=(-1)^{n} D_{n-1}^{T}$ we get

$$
\left[\begin{array}{cc}
I & -(k / \mu) M_{n-1}^{-1} D_{n-1}^{T} \\
D_{n-1} & 0
\end{array}\right]\left[\begin{array}{l}
f \\
p
\end{array}\right]=\left[\begin{array}{c}
0 \\
\phi \omega
\end{array}\right] .
$$

Assuming that the domain has only one connected component, the pressure is unique only up to a constant. Hence, pressure at a single point must be fixed to an arbitrary value to get a unique solution. To impose the boundary conditions the right hand side of equation (4.5) is adjusted for the known boundary fluxes. Such an adjustment is also done for the assumed pressure at one point. This is a standard procedure and it is described here briefly for completeness. The adjustments are done by taking the linear combination of the columns of the linear system matrix in (4.5) corresponding to the known $f$ and $k$. The coefficients in the linear combination are the known $f$ and $k$ values. The result is subtracted from the right hand side. These columns and corresponding rows are then deleted from the matrices in equation (4.5).

Equation (4.5) can be written in a simpler standard saddle point form [8] by starting from equation (3.11) instead of (3.8). This is equivalent to multiplying the first block row of $(4.5)$ by $-(\mu / k) M_{n-1}$ and the resulting system is

$$
\left[\begin{array}{cc}
-(\mu / k) M_{n-1} & D_{n-1}^{T} \\
D_{n-1} & 0
\end{array}\right]\left[\begin{array}{l}
f \\
p
\end{array}\right]=\left[\begin{array}{c}
0 \\
\phi \omega
\end{array}\right] .
$$

To take into account the boundary conditions (4.3) and the assumed pressure at a point, the adjustments described above are applied to equation (4.6). The matrix on the left hand side of (4.6) is a saddle type matrix of the form

$$
\left[\begin{array}{cc}
A & B^{T} \\
B & 0
\end{array}\right]
$$

Even after the boundary fluxes and assumed pressure are taken into account the form of the matrix stays the same, using sub-matrices of $A$ and $B$. Here the matrix $A$ is a diagonal matrix with nonzero diagonal entries since $A=(-\mu / k) M_{n-1}$ and $M_{n-1}$ is the diagonal discrete Hodge matrix. One difference between our method and Raviart-Thomas finite elements is that in the latter case $A$ is not a diagonal matrix. 
The system obtained by adjusting equations (4.5) or (4.6) for boundary conditions and assumed pressure at a point can be solved for the remaining pressures and the unknown fluxes by using any of the standard techniques for saddle type matrices [8]. For the results in this paper we used the Schur complement reduction method [8]. In our case it is particularly simple since the $A$ matrix can be explicitly inverted trivially.

4.1. Flux visualization. Once the flux has been determined, we visualize it by using Whitney interpolation as described in Section 2.6 to get a smooth $(n-1)$-form inside each $n$-simplex. We then obtain the corresponding velocity vector fields sampled at barycenters. The sampling could be done at any location or locations in the interior of the $n$-simplices, not just at the barycenter. Given a flux value, we can determine the velocity as follows. The flux $f$ is related to the velocity by $f=*\left(v^{b}\right)$ which implies that $* f=* *\left(v^{b}\right)=(-1)^{n-1} v^{b}$.

Consider first $n=2$ and let the value of the Whitney interpolated flux 1 -form at a sampling point be $a d x+b d y$ where $a$ and $b$ are some constants. Then

$$
v^{b}=-(* f)=-*(a d x+b d y)=b d x-a d y,
$$

which implies that for standard metric in $\mathbb{R}^{2}$, the velocity $v$ at that point is the vector $(b,-a)$. For $n=3$ if the value of the Whitney interpolated flux 2 -form at a sampling point inside a tetrahedron is $f=a d y \wedge d z+b d z \wedge$ $d x+c d x \wedge d y$ then the associated velocity is given by

$$
v^{b}=* f=*(a d y \wedge d z+b d z \wedge d x+c d x \wedge d y)=a d x+b d y+c d z,
$$

which implies that for standard metric in $\mathbb{R}^{3}$, the velocity $v$ is the vector $(a, b, c)$.

\section{Heterogeneous Permeability and Hodge Star}

In many physical problems the Hodge star (which is an operator depending on the metric) appears as a material dependent operator. For example when Maxwell's equations are written in terms of differential forms, the electric permittivity and the magnetic permeability are both Hodge stars [15]. The permittivity Hodge star relates the electric field 1-form to the electric induction 2-form and the permeability Hodge star relates the magnetic flux density 2-form to the magnetic field 1-form.

In this section we rewrite the flux form of Darcy's law (equation (3.8)) in a form that permits its discretization when the permeability is a spatially dependent scalar quantity. The scalar permeability value is discretized as constant in each $n$-simplex. From these scalar values of the permeability, a spatially dependent discrete Hodge star operator is constructed. This involves combining the scalar permeabilities across an $(n-1)$-simplex in a weighted average that is suggested by DEC and described in this section. The resulting matrix for this heterogeneous Hodge star is still a diagonal matrix. 
We start with the equations $(3.8)-(3.10)$ with the exception that the term $(k / \mu)(* \mathrm{~d} p)$ is replaced by $(1 / \mu)\left(*^{k} \mathrm{~d} p\right)$. Thus Darcy law (3.8) now becomes

$$
f+\frac{1}{\mu}\left(*^{k} \mathrm{~d} p\right)=0 \quad \text { in } \Omega
$$

where the Hodge star operator $*^{k}$ is the spatially dependent Hodge star that depends on the permeability of the medium. The continuity equation and boundary condition stay the same as equations (3.9) and (3.10). This heterogeneous Hodge star is discretized as a diagonal matrix where the diagonal entry corresponding to an $(n-1)$-simplex $\sigma^{n-1}$ in the matrix $\left(M_{n-1}^{k}\right)^{-1}$ is

$$
\frac{\left|\sigma^{n-1}\right|}{\left|\star \sigma^{n-1}\right|} \frac{k_{+}\left|\star \sigma^{n-1} \cap \sigma_{+}^{n}\right|+k_{-}\left|\star \sigma^{n-1} \cap \sigma_{-}^{n}\right|}{\left|\star \sigma^{n-1}\right|} .
$$

Here $\sigma_{+}^{n}$ and $\sigma_{-}^{n}$ are the two simplices that contain $\sigma^{n-1}$, and $k_{+}$and $k_{-}$are the permeabilities in these $n$-simplices. The dual edge $\star \sigma^{n-1}$ points from $\sigma_{-}$into $\sigma_{+}$. The expression $\left|\star \sigma^{n-1} \cap \sigma_{+}^{n}\right|$ stands for the length of the portion of dual edge $\star \sigma^{n-1}$ that lies in $\sigma_{+}^{n}$ etc. For $k_{+}=k_{-}=k$ expression (5.2) reduces to

$$
k \frac{\left|\sigma^{n-1}\right|}{\left|\star \sigma^{n-1}\right|},
$$

which is the corresponding diagonal entry of $M_{n-1}^{-1}$, thus yielding the usual discretization of the homogeneous Hodge star scaled by $k$.

The ratio on the right in expression (5.2) can be interpreted as a weighted average of permeabilities. Note that it is not a simple arithmetic or geometric mean of the permeabilities. The weights are the same as the ones that were used for averaging piecewise constant vector fields along a shared face in [28]. In [28] it was shown that these are the unique weights that yield a discrete divergence theorem. See [28, Figure 5.4, Section 5.5 and Section 6.1] for more details.

\section{Numerical Results}

We illustrate the performance of the proposed DEC based numerical method for Darcy flow using many standard test problems. In all the figures here that show a velocity vector field, the flux $f$ has been visualized as the corresponding vector field. As described in Section 4.1 the velocity vector field $v$ is obtained from $f$ by using the relationship $v^{b}=(-1)^{n-1} * f$. The velocity vector field is sampled at barycenters of top dimensional simplices and displayed as arrows based at barycenters. The pressure in most figures is displayed by plotting it against the $x$ coordinate of the circumcenter which is where the pressures are defined. The only exceptions are the pressure plots in Figure 6 in which the pressure is displayed by coloring the triangle with a single color based on pressure value at the circumcenter. Figures 4, 5, 6, 10 and 11 were constructed from data generated by a Python implementation of our numerical method that used the PyDEC software [7]; 
and Figures 7, 8 and 9, were generated by a MATLAB implementation of our numerical method.

6.1. Patch tests in 2D. The first results shown in Figure 4 are for patch tests [31]. It is desirable that for simple meshes a numerical method for Darcy flow should reproduce constant velocity and linear pressure exactly up to machine precision. The boundary condition in these tests is derived from a constant horizontal velocity $(1,0)$. Thus, for example, in the square domain $v \cdot \hat{n}=\psi=-1$ on the left edge, $\psi=1$ on the right edge, and 0 on the top and bottom edges of the square. Keeping in mind the orientations and the definition of $\gamma$, when the discretized equations (4.1)-(4.3) are used, $f=1$ on the left and right edges and 0 on the top and bottom edges of the square.

We now explain the sign of $f$ in more detail. Suppose the bottom left and top left corner vertices of the square domain in Figure 4 are labeled $v_{0}$ and $v_{1}$ and the edge between them is oriented from $v_{0}$ to $v_{1}$. We will use the name $\sigma$ for this oriented edge $\left[v_{0}, v_{1}\right]$ and denote the vector from $v_{0}$ to $v_{1}$ by $\vec{\sigma}$. Assume also that the square, and hence the triangle to which $\sigma$ belongs, is oriented counterclockwise. We use the same name $f$ for the 1-cochain $f$ of equations (4.1)-(4.3) and the 1-form $f$ of equations (3.8)-(3.10), but to be more precise the cochain $f$ should be referred to as $R(f)$ where $R$ is the de Rham map of Section 2.4. The following calculation explains why $\langle R(f), \sigma\rangle=+1$ in this setting.

$$
\begin{aligned}
\langle R(f), \sigma\rangle & =\int_{\sigma} \psi \gamma=\int_{\sigma}(v \cdot \hat{n}) \gamma=(v \cdot \hat{n}) \int_{\sigma} \gamma \\
& =(-1) \int_{\sigma} \gamma=-\gamma(\vec{\sigma})=-\omega(\hat{n}, \vec{\sigma})=+1 .
\end{aligned}
$$

Here the third equality follows from the fact that $v \cdot \hat{n}$ is constant along $\sigma$, the fifth equality is true because $\sigma$ is a straight line, and $\omega(\hat{n}, \vec{\sigma})=-1$ because the length of $\sigma$ is 1 and the basis $(\hat{n}, \vec{\sigma})$ for the plane is oriented clockwise, which is opposite of the orientation of the square. If $\sigma$ had been oriented from $v_{1}$ to $v_{0}$ instead, the value of $\langle R(f), \sigma\rangle$ would have been -1 instead.

In this test, we are also given that $\phi=0$, so that $\operatorname{div} v=0$ (equivalently, $\mathrm{d} f=0)$ in the domain. The parameters $k$ and $\mu$ are 1 , and $g=0$ so there is no external forcing. This example is constructed by starting with pressure $p=-x+c$ for some constant $c$. Then it follows that $v=(1,0)$ everywhere and $\operatorname{div} v=0$ as given. The numerical method is given the $\phi$ and $\psi$ and the pressure and flux is computed using the method. Although such patch tests do not guarantee that a method is high quality [6], it is a convenient way to find problems with methods, as shown in Figure 1. If a method fails such a simple test, it is probably unsuitable for the problem.

The relative errors for the nonzero pressures are less than $3 \times 10^{-16}$ for the square and less than $7 \times 10^{-16}$ for the hexagon shown in Figure 4 . Thus 
for these simple meshes the relative error is close to machine precision. The same test is repeated for a larger mesh of a square domain in Figure 5 for which the relative error in pressure is less than $9 \times 10^{-12}$.

6.2. Known solution with nonzero source term. In the next test we compare the solution computed using our method with an analytically known solution. Again the parameters $k$ and $\mu$ are 1 and $g=0$. The solution is constructed by starting with pressure $p=\cos (\pi x) \cos (\pi y)$ from which an expression for $v$ is derived. From $v$ one computes the divergence to derive that the source/sink term is $\phi=2 \pi^{2} \cos (\pi x) \cos (\pi y)$. The boundary data $\psi$ is constructed from the analytically computed $v$. The numerical method is given $\phi$ and $\psi$ and used to compute $v$ and $k$ in the domain from that. Figure 6 shows a comparison of the computed pressure and velocity with the analytical solution.

6.3. Discontinuous permeability. One of the new results of our approach is a Hodge star that allows the discretization of the equations in the case of spatially varying scalar permeability. The permeability is taken to be constant in each $n$-simplex. The resulting heterogeneous diagonal Hodge star matrix was defined in Section 5. An important aspect of any numerical method for Darcy flow is how well it can address discontinuities in permeability. Our heterogeneous discrete Hodge star allows us to test this aspect of our method. Figure 7 shows the results of a patch test with discontinuous permeability in two adjoining domains. The domain is a rectangle in which the triangles in the left half are given a permeability of $k_{1}$ and those in the right half are given a permeability of $k_{2}$. The fluid flows in from the left and exits from the right. The pressure should be a piecewise linear function whose slope depends on the permeability and the velocity in the domain should be constant. This is demonstrated in the results from our method.

6.4. Layered medium. Another common test in the Darcy flow literature is when the discontinuities in the permeability vary across the flow rather than along it. Such a medium is typically called a layered medium in which the various layers are given different permeabilities. In our layered medium computation we perform two tests. In one the permeabilities alternate between 1 and 5 and in another they alternate between 1 and 10. The fluid comes into the domain from the left and exits from the right as in our patch tests. The velocity should be horizontal and constant in a layer. It should be larger in the low permeability layers as computed by our method and shown in Figure 8. The correct pressure profile should be a linear function of $x$ and this is seen in Figure 9 .

6.5. Patch tests in 3D. Many numerical formulations perform well in $2 \mathrm{D}$, but their natural extensions to 3D do not perform well. For example, the famous Raviart-Thomas element passes patch test in 2D. However, it is well-known that this element does not pass patch in $3 \mathrm{D}$ for distorted meshes 
[38], and hence is not suitable for three-dimensional numerical simulations on unstructured meshes.

Herein we show that the proposed formulation performs well even in 3D. To illustrate this we considered two different computational domains, which are shown in Figures 10 and 11. The first domain is a polyhedron with 16 tetrahedra and the second domain is a cube with 244 tetrahedra. In both cases the circumcenter of each tetrahedron is in its interior. In addition, all the triangles in the cube mesh are also acute angled (this is an example of a completely well-centered mesh [46]). Note that the elements in these meshes are distorted. The analytical solution is constant velocity along $x$ direction, and pressure linearly varying along $x$ direction. The obtained numerical results are plotted in Figures 10 and 11 which shows that the numerical method performed well. For example, the latter figure shows that the relative error in pressure is less than $2 \times 10^{-13}$.

6.6. Circumcenter versus barycenter. One of the main focuses of this paper is on structure preserving numerical methods, generated by a systematic application of DEC. The proposed method has both local and global mass balance properties. In addition, we show that the proposed method can also exactly represent linear variation of pressure within a domain. We also highlight the need for careful choice of locations for the pressure to get better numerical solutions. DEC naturally provides the locations of pressure that conserve local and global mass balance, and also exactly represent linear variation of pressure. In DEC, for each element, pressure is located at circumcenter. The fact that each $(n-1)$-simplex and its circumcentric dual edge are orthogonal is important here. One common choice used in the literature as location points for pressure are barycenters. In Figure 3 we show that choice of barycenter cannot exactly represent linear variation of pressure (along with local and global mass balance) whereas location of pressure at circumcenter can. This also illustrates how a geometrical view of PDEs can provide good insights into developing novel structure preserving numerical methods.

\section{Conclusions and Future Work}

Our goal in this paper has been to introduce a numerical method for Darcy flow derived using discrete exterior calculus (DEC). We have shown that this approach results in a unified derivation of methods for both two- and threedimensions. We have also demonstrated the numerical performance of these methods. For example, the method is shown to pass several patch and other standard test problems in both two and three dimensions. Our DEC based approach is intrinsic, in the sense that it involves quantities and operations that do not depend on how the mesh is embedded in $\mathbb{R}^{3}$. As a result, it should be easy to use the method for Darcy flow on a curved surface using a well-centered discretization of such a surface. This is one avenue for future research. 


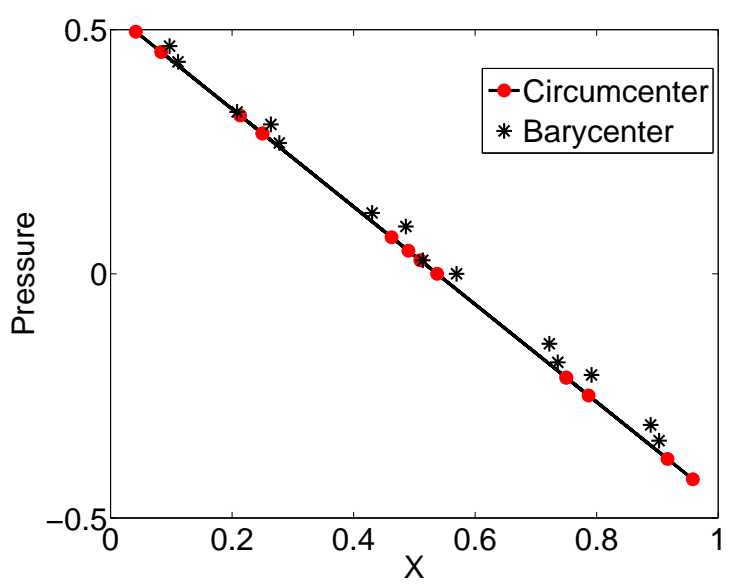

Figure 3: Locating pressures at barycenters does not work. Shown here is the pressure computed for a square domain in which the boundary condition is given by constant horizontal velocity pointing to right. Thus velocity in the domain should be constant and pressure linear which is reproduced when pressures are located at circumcenters as dictated by DEC.

Even in the case of domains that are open subsets of $\mathbb{R}^{2}$ or $\mathbb{R}^{3}$ the required computations such as circumcenter calculation, and computation of volumes, areas and lengths, can be built from linear algebra operations which simplifies the implementation as demonstrated in [7].

The philosophy of DEC is to discretize the operators and objects in such a way that the their properties in smooth calculus have discrete analogs. As a result, for example, in our method mass balance is satisfied both locally (that is, in each element) and globally because of the way the fluxes are represented and because Stokes' theorem is true by definition in DEC.

A pedagogical advantage of the DEC approach is that once the language of exterior calculus has been mastered and the discretizations understood, the translation from a PDE to its corresponding discrete equation, and from there to the matrix form are trivial steps. Compare for example the smooth equation (3.8), the corresponding discrete equation (4.1) and the first block row of the matrix equation (4.5). There is also a clean separation in the matrix form equation (4.6) between the parts depending only on the mesh connectivity (the top right and bottom left blocks) and that depending on how the physical variable are related (the top left part) which usually involves constitutive relationships as well. These depend on material measurements, and hence the inaccuracies of measurement of material properties does not corrupt that part of the matrix that is topological. In this aspect our method shares this good property of some mixed finite element method formulations. 
In addition to applying DEC to Darcy flow, in this paper we have also developed a discretization of Hodge star for non-homogeneous medium. We used this in examples in which the permeability varies across the mesh, either along the flow or transversal to the flow. This discretization of a spatially varying Hodge star should be useful in other PDEs as well.

There are many avenues for future research even within Darcy flow. Flow on curved surfaces has already been mentioned before. Another direction for further work is the proper DEC discretization of anisotropic permeability. It is also possible to remove the requirement of using well-centered triangulations and this needs to be explored further too. Finally the numerical analysis of convergence and stability properties remains to be done.

\section{ACKNOWLEDGMENTS}

The research of ANH and JHC was supported by the National Science Foundation with an NSF CAREER Award (Grant No. DMS-0645604). The research of KBN was supported in part by the Department of Energy through a SciDAC-2 project (Grant No. DOE DE-FC02-07ER64323). ANH would like to thank his student Evan VanderZee for help in creating many of the well-centered meshes in this paper. The authors would like to thank Prof. Albert Valocchi and Prof. Arif Masud for valuable discussions. The opinions expressed in this paper are those of the authors and do not necessarily reflect that of the sponsors.

\section{REFERENCES}

[1] Abraham, R., Marsden, J. E., and Ratiu, T. Manifolds, Tensor Analysis, and Applications, second ed. Springer-Verlag, New York, 1988.

[2] Achdou, Y., Bernardi, C., and Coquela, F. A priori and a posteriori analysis of finite volume discretizations of Darcy's equations. Numerische Mathematik 96 (2003), 17-42.

[3] Arnold, D. N., Bochev, P. B., Lehoucq, R. B., Nicolaides, R. A., And Shashkov, M., Eds. Compatible Spatial Discretizations, vol. 142 of The IMA Volumes in Mathematics and its Applications. Springer New York, 2006. doi:10.1007/0-387-38034-5.

[4] Arnold, D. N., Falk, R. S., And Winther, R. Finite element exterior calculus, homological techniques, and applications. In Acta Numerica, A. Iserles, Ed., vol. 15. Cambridge University Press, 2006, pp. 1155. URL http://www.ima. umn. edu/ arnold/papers/acta.pdf.

[5] Arnold, V. I. Mathematical Methods of Classical Mechanics, second ed. Springer-Verlag, New York, 1989. Translated from the Russian by K. Vogtmann and A. Weinstein. 
[6] Babuska, I., And Narasimhan, R. The Babuska-Brezzi condition and the patch test: an example. Computer Methods in Applied Mechanics and Engineering 140, 1-2 (January 1997), 183-199. doi: 10.1016/S0045-7825(96)01058-4.

[7] Bell, N., And Hirani, A. N. PyDEC: Algorithms and software for Discrete Exterior Calculus. In preparation., 2008.

[8] Benzi, M., Golub, G. H., And Liesen, J. Numerical solution of saddle point problems. Acta Numerica 14 (2005), 1-137.

[9] Bochev, P., And Dohrmann, C. A computational study of stabilized, low-order $C^{0}$ finite element approximations of Darcy equations. Computational Mechanics 38, 4-5 (2006), 323-333. doi:10. 1007/s00466-006-0036-y.

[10] Bochev, P. B., And Hyman, J. M. Principles of mimetic discretizations of differential operators. In Compatible Spatial Discretizations, D. N. Arnold, P. B. Bochev, R. B. Lehoucq, R. A. Nicolaides, and M. Shashkov, Eds., vol. 142 of The IMA Volumes in Mathematics and its Applications. Springer, Berlin, 2006, pp. 89-119. doi: 10. 1007/0-387-38034-5.

[11] Bossavit, A. Mixed finite elements and the complex of Whitney forms. In The Mathematics of Finite Elements and Applications VI, J. Whiteman, Ed. Academic Press, 1988, pp. 137-144.

[12] Bossavit, A. A rationale for "edge elements" in 3-D fields computations. IEEE Trans. Mag. 24, 1 (January 1988), 74-79.

[13] Bossavit, A. Whitney forms : A class of finite elements for threedimensional computations in electromagnetism. IEE Proceedings 135, Part A, 8 (November 1988), 493-500.

[14] Bossavit, A. Computational Electromagnetism : Variational Formulations, Complementarity, Edge Elements. Academic Press, 1998.

[15] Bossavit, A. On the geometry of electromagnetism (4): Maxwell's house. Journal of the Japan Society of Applied Electromagnetics 6, 4 (1998), 318-326.

[16] Braess, D. Finite Elements: Theory, Fast Solvers, and Applications in Solid Mechanics, third ed. Cambridge University Press, Cambridge, 2007. Translated from the 1992 German edition by Larry L. Schumaker.

[17] Brezzi, F., Douglas, J., And Marini, L. D. Two families of mixed finite elements for second order elliptic problems. Numerische Mathematik 47, 2 (June 1985), 217-235. doi:10.1007/BF01389710.

[18] BrezzI, F., AND Fortin, M. Mixed and hybrid finite element methods, volume 15 of Springer series in computational mathematics. SpringerVerlag, New York, 1991.

[19] Chen, Z. Finite Element Methods and Their Applications. Scientific Computation. Springer, 2005.

[20] Chou, S., And Vassilevski, P. A general mixed covolume framework for constructing conservative schemes for elliptic problems. Mathematics of Computation 68, 227 (1999), 991-1011. 
[21] Chou, S.-H., Kwak, D. Y., And Vassilevski, P. S. Mixed covolume methods for elliptic problems on triangular grids. SIAM Journal on Numerical Analysis 35, 5 (1998), 1850-1861. URL http://www. jstor. org/stable/2587277.

[22] Desbrun, M., Hirani, A. N., Leok, M., and Marsden, J. E. Discrete exterior calculus. arXiv:math.DG/0508341 (August 2005). URL http://arxiv.org/pdf/math.DG/0508341.

[23] Desbrun, M., Kanso, E., And Tong, Y. Discrete differential forms for computational modeling. In Discrete Differential Geometry, A. I. Bobenko, J. M. Sullivan, P. Schröder, and G. M. Ziegler, Eds., vol. 38 of Oberwolfach Seminars. Birkhäuser Basel, 2008, pp. 287-324. doi: 10.1007/978-3-7643-8621-4_16.

[24] Dodziuk, J. Finite-difference approach to the Hodge theory of harmonic forms. Amer. J. Math. 98, 1 (1976), 79-104.

[25] Elcott, S., Tong, Y., Kanso, E., Schröder, P., And Desbrun, M. Stable, circulation-preserving, simplicial fluids. ACM Transactions on Graphics 26, 1 (2007), 4. doi:http://doi .acm.org/10.1145/ 1189762.1189766.

[26] Erten, H., AND Üngör, A. Computing acute and non-obtuse triangulations. In Proceedings of the 19th Canadian Conference on Computational Geometry (CCCG2007) (August 20-22 2007).

[27] Hildebrandt, K., Polthier, K., and Wardetzky, M. On the convergence of metric and geometric properties of polyhedral surfaces. Geometriae Dedicata 123, 1 (December 2006), 89-112. doi:10.1007/ s10711-006-9109-5.

[28] Hirani, A. N. Discrete Exterior Calculus. PhD thesis, California Institute of Technology, May 2003. URL http://resolver.caltech. edu/CaltechETD : etd-05202003-095403.

[29] Hughes, T. J. R., Masud, A., And Wan, J. A stabilized mixed discontinuous Galerkin method for Darcy flow. Computer Methods in Applied Mechanics and Engineering 195 (2006), 3347-3381.

[30] Hyman, J. M., And Shashkov, M. Natural discretizations for the divergence, gradient, and curl on logically rectangular grids. Comput. Math. Appl. 33, 4 (1997), 81-104.

[31] Irons, B., And LoikKanen, M. An engineers' defence of the patch test. International Journal for Numerical Methods in Engineering 19, 9 (1983), 1391-1401. doi:10.1002/nme.1620190908.

[32] Lipnikov, K., Shashkov, M., and Svyatskiy, D. The mimetic finite difference discretization of diffusion problem on unstructured polyhedral meshes. Journal of Computational Physics 211, 2 (January 2006), 473-491. doi:10.1016/j.jcp.2005.05.028.

[33] Lipnikov, K., Shashkov, M., Svyatskiy, D., and Vassilevski, Y. Monotone finite volume schemes for diffusion equations on unstructured triangular and shape-regular polygonal meshes. Journal of Computational Physics 227, 1 (492-512 2007). doi:10.1016/j.jcp.2007.08. 
008.

[34] Maenara, H. Acute triangulations of polygons. European Journal of Combinatorics 23, 1 (2002), 45-55.

[35] Masud, A., And Hughes, T. J. R. A stabilized mixed finite element method for Darcy flow. Computer Methods Applied Mechanics and Engineering 191 (2002), 4341-4370. doi:10.1016/S0045-7825(02) 00371-7.

[36] Munkres, J. R. Elements of Algebraic Topology. Addison-Wesley Publishing Company, Menlo Park, 1984.

[37] Nakshatrala, K. B., Masud, A., and Huelmstad, K. D. On finite element formulations for nearly incompressible linear elasticity. Computational Mechanics 41 (2008), 547-561.

[38] Nakshatrala, K. B., Turner, D. Z., Huelmstad, K. D., And MAsud, A. A mixed stabilized finite element formulation for Darcy flow based on a multiscale decomposition of the solution. Computer Methods in Applied Mechanics and Engineering 195 (2006), 4036-4049.

[39] Nedelec, J. C. Mixed finite elements in $\mathbb{R}^{3}$. Numerische Mathematik 35, 3 (1980), 315-341. doi:10.1007/BF01396415.

[40] Nicolaides, R. A. Direct discretization of planar div-curl problems. SIAM Journal on Numerical Analysis 29, 1 (1992), 32-56. URL http: //www.jstor.org/stable/2158074.

[41] Nicolaides, R. A., AND Wu, X. Covolume solutions of threedimensional div-curl equations. SIAM Journal on Numerical Analysis 34, 6 (1997), 2195-2203.

[42] Perot, J., And Subramanian, V. Discrete calculus methods for diffusion. Journal of Computational Physics 224, 1 (2007), 59-81. doi: $10.1016 / \mathrm{j} \cdot \mathrm{jcp} .2006 .12 .022$.

[43] Raviart, P.-A., and Thomas, J. M. A mixed finite element method for 2nd order elliptic problems. In Mathematical aspects of finite element methods (Proc. Conf., Consiglio Naz. delle Ricerche (C.N.R.), Rome, 1975), Lecture Notes in Math., Vol. 606. Springer, Berlin, 1977, pp. 292315.

[44] Vanderzee, E., Hirani, A. N., And Guoy, D. Triangulation of simple 3d shapes with well-centered tetrahedra. Tech. Rep. UIUCDCSR-2008-2970, Department of Computer Science, University of Illinois at Urbana-Champaign, 2008. Also available as a preprint at arXiv as arXiv:0806.2332v1 [cs.CG]. URL http://arxiv.org/abs/0806.2332.

[45] Vanderzee, E., Hirani, A. N., Guoy, D., and Ramos, E. Wellcentered planar triangulation - An iterative approach. In Proceedings of 16th International Meshing Roundtable (Seattle, Washington, October 14-17 2007), M. L. Brewer and D. Marcum, Eds., Springer Berlin, pp. 121-138. doi:10.1007/978-3-540-75103-8.

[46] Vanderzee, E., Hirani, A. N., Guoy, D., and Ramos, E. 
Well-centered triangulation. Tech. Rep. UIUCDCS-R-2008-2936, Department of Computer Science, University of Illinois at UrbanaChampaign, February 2008. Also available as a preprint at arXiv as arXiv:0802.2108v1 [cs.CG]. URL http://arxiv.org/abs/0802.2108.

[47] Wardetzky, M. Convergence of the cotangent formula: An overview. In Discrete Differential Geometry, A. I. Bobenko, J. M. Sullivan, P. Schröder, and G. M. Ziegler, Eds., vol. 38 of Oberwolfach Seminars. Birkhäuser Basel, 2008, pp. 275-286. doi:10.1007/ 978-3-7643-8621-4_15.

[48] Whitney, H. Geometric Integration Theory. Princeton University Press, Princeton, N. J., 1957.

[49] Yuan, L. Acute triangulations of polygons. Discrete and Computational Geometry 34, 4 (2005), 697-706.

Correspondence to: Professor Anil N. Hirani, Department of Computer Science, University of Illinois at Urbana-Champaign, 201 N. Goodwin Ave., URBANA, IL 61801.

E-mail address: hirani@cs.uiuc.edu

URL: http://www.cs.uiuc.edu/hirani

Dr. Kalyana Babu Nakshatrala, Department of Civil and Environmental ENGINEERING, 2524 Hydrosystems LABORATORY, UNiversity of ILlinOIS AT URBANAChampaign, Urbana, IL 61801.

E-mail address: nakshatr@uiuc.edu

Jehanzeb H. Chaudhry, Department of Computer Science, University of Illinois at Urbana-Champaign, 201 N. Goodwin Ave., Urbana, IL 61801.

E-mail address: jhameed2@uiuc.edu 

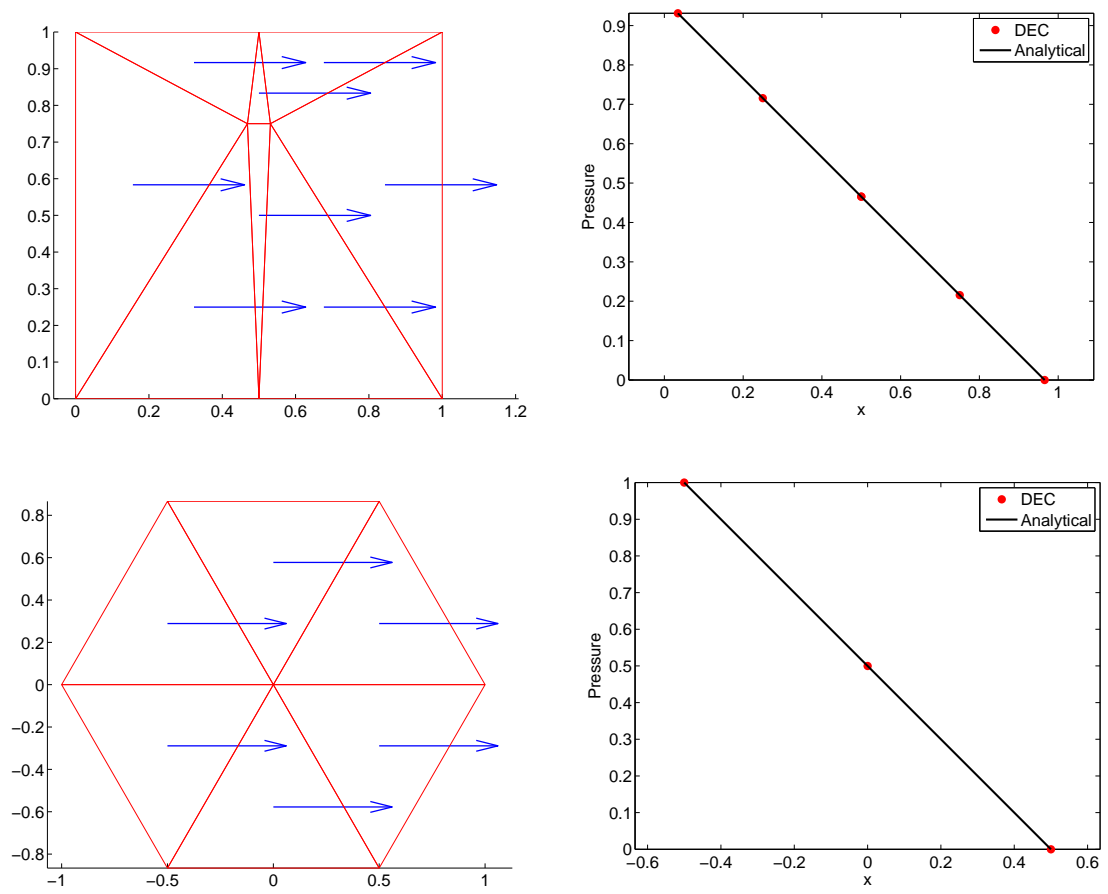

Figure 4: Results from patch tests. The boundary conditions are obtained from a velocity of 1 in positive $x$ direction. Thus, for the square domain, in equation (3.3), $\psi=-1$ on left edge, 1 on right edge, and 0 on top and bottom edges. For both the square and hexagon domains it is also given is that $\phi=0$ in (3.2), constants $k=1$, $\mu=1$ and external body force acceleration $g=0$ in (3.1). The pressure should be linear as shown in the right figures. The left figures show that the velocity is constant, as expected. The velocity displayed is interpolated from the flux through each edge, using Whitney 1-form and sampled and converted to a vector. 


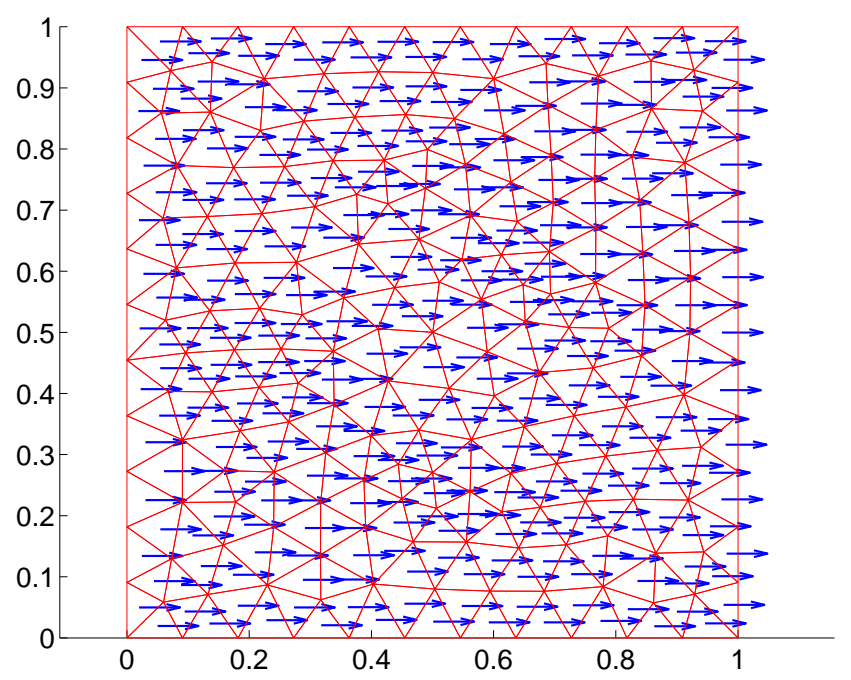

Figure 5: Patch test with larger mesh and with the same boundary conditions, parameters and other data as the square in Figure 4. 

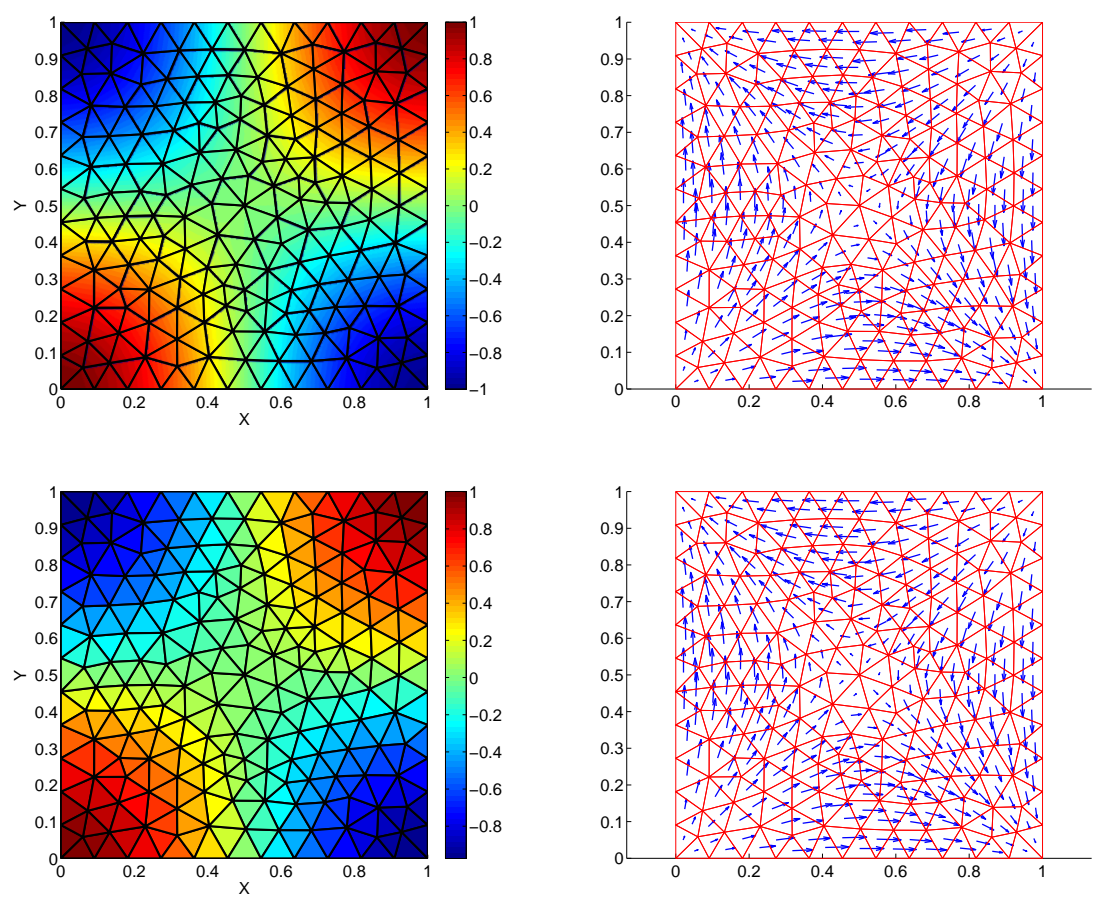

Figure 6: Comparison of analytically known solution with a solution computed using the method developed here. The top left figure shows the pressure part of the analytical solution to the Darcy law problem with $k=1, \mu=1, g=0$, and $\phi=2 \pi^{2} \cos (\pi x) \cos (\pi y)$. This implies $p=\cos (\pi x) \cos (\pi y)$ which is shown overlaid on the mesh. In the left bottom figure we show the pressure computed with the numerical method proposed here. Each triangle is colored by the pressure at its circumcenter. The top right figure shows the analytically computed vector field, and the numerically computed vector field is shown in the right bottom figure, which is obtained from the flux by interpolation of 1-forms. 

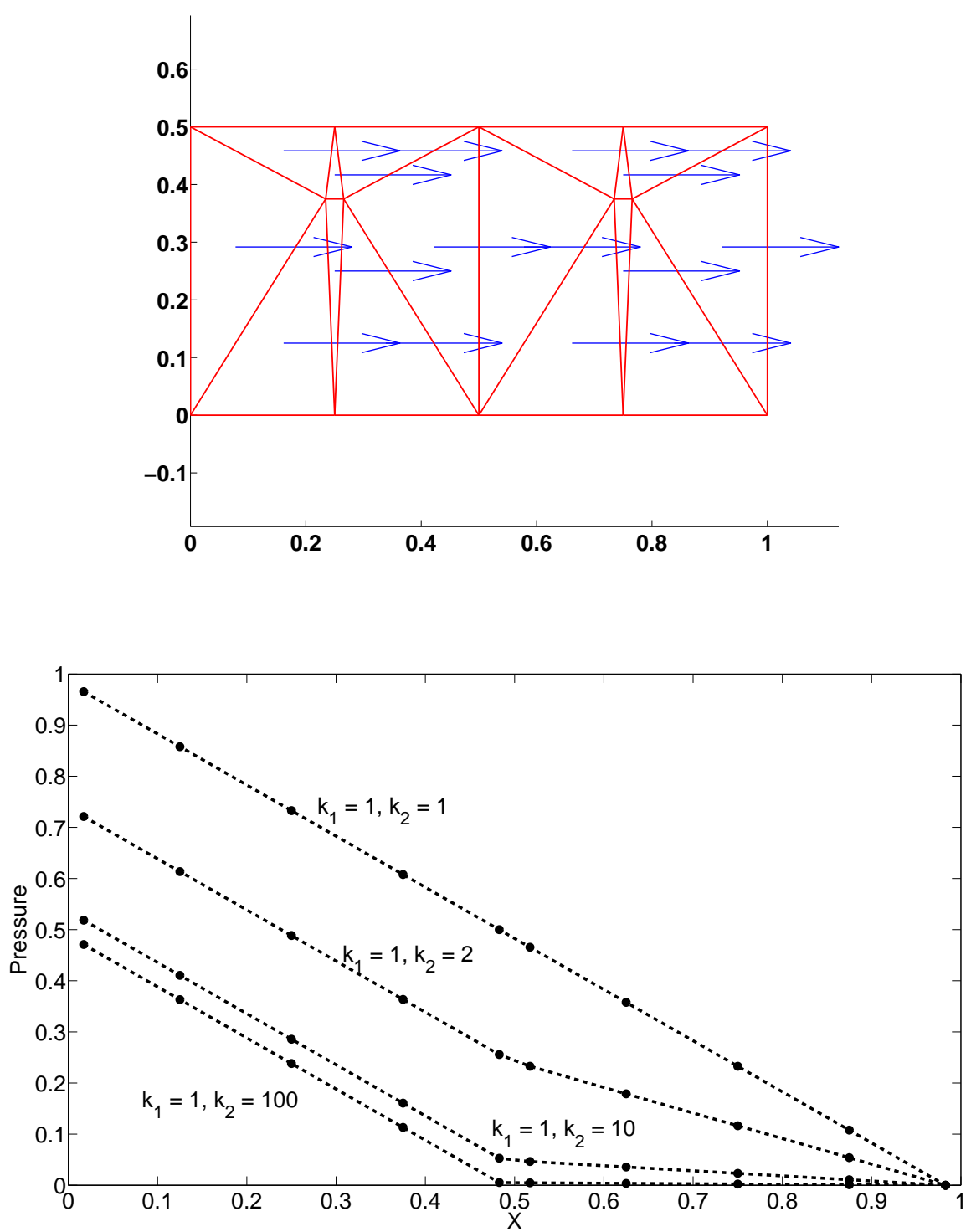

Figure 7: In this test the left and right halves of the mesh are given different permeabilities, $k_{1}$ on the left and $k_{2}$ on right. Thus the permeability jumps across the middle vertical edge at $x=0.5$. The values that we use for $\left(k_{1}, k_{2}\right)$ are $(1,1)$, $(1,2),(1,10)$ and $(1,100)$. The boundary condition is derived from velocity $(1,0)$ as in Figure 4. The top figure shows the computed velocity interpolated from flux for $k_{1}=1$ and $k_{2}=10$. All other value pairs also result in a constant velocity solution as expected. The pressure is piecewise linear with a jump at the discontinuity at $x=0.5$ as shown in the bottom figure. 

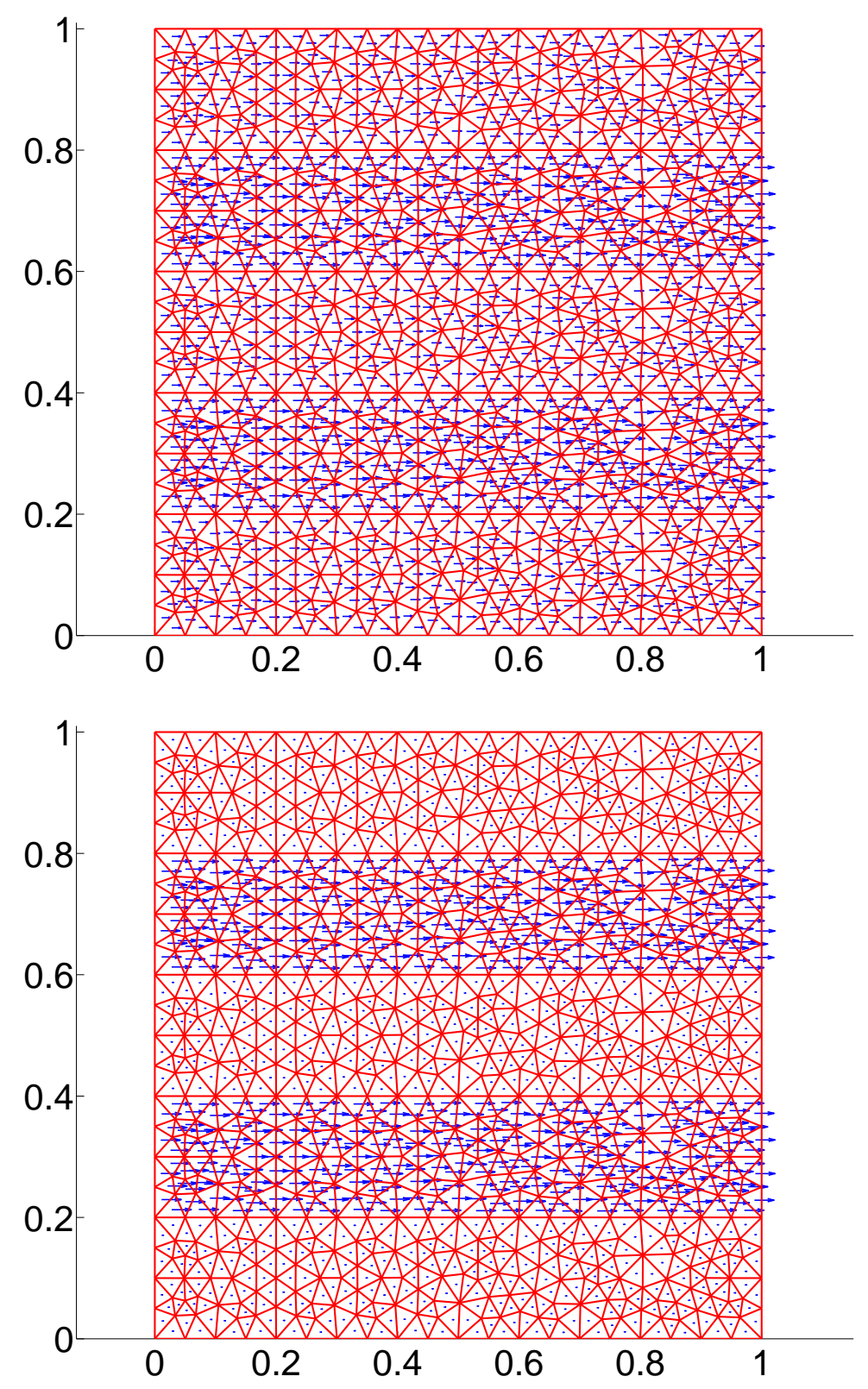

Figure 8: Layered medium with 2 different permeability patterns. The domain has 5 layers with alternating permeability. In the top figure the permeability $k$, from bottom layer to top is $5,10,5,10$ and 5 . In the bottom figure the permeability $k$ is $1,10,1,10$ and 1 . The computed flux is visualized as a vector field. 

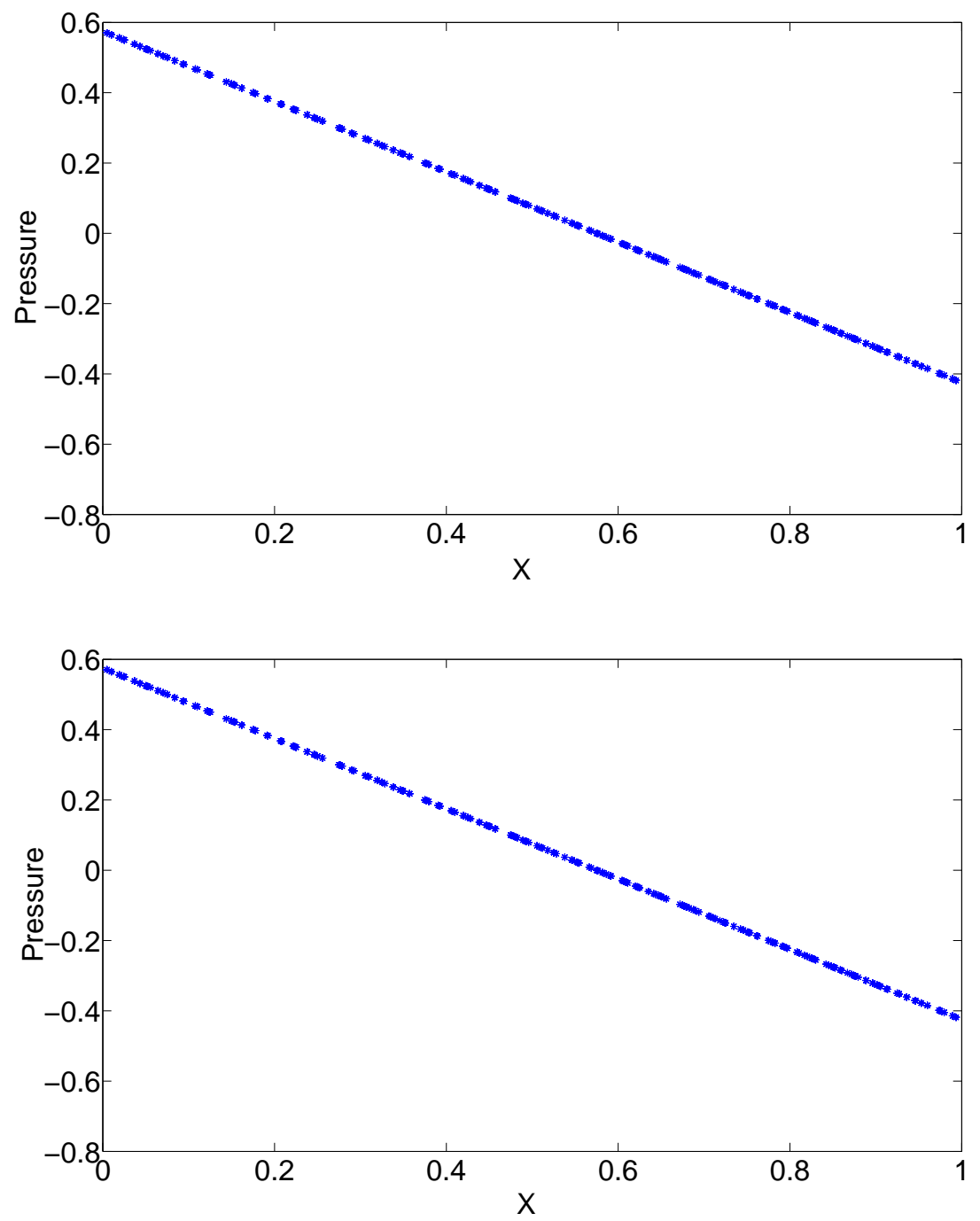

Figure 9: Pressure for the layered medium show in Figure 8. The pressure is linear as expected. The top and bottom figures correspond to the top and bottom figures in Figure 8. 

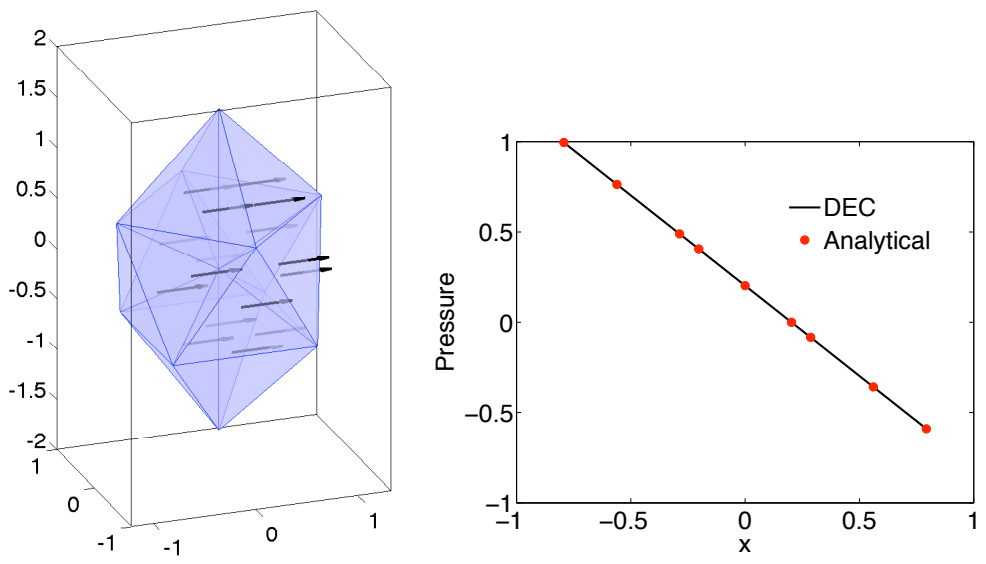

Figure 10: Patch test in 3D. The mesh shown has 16 tetrahedra, each of which is well-centered. Each triangle in the mesh is also well-centered. The fluid velocity on the boundary is from negative $x$ to positive $x$ direction, with no $y$ or $z$ components. The fluxes (2-cochains) across the internal faces are computed using our method and interpolated using the Whitney map. This is then sampled at the circumcenters, converted into a vector field and plotted as arrows. 

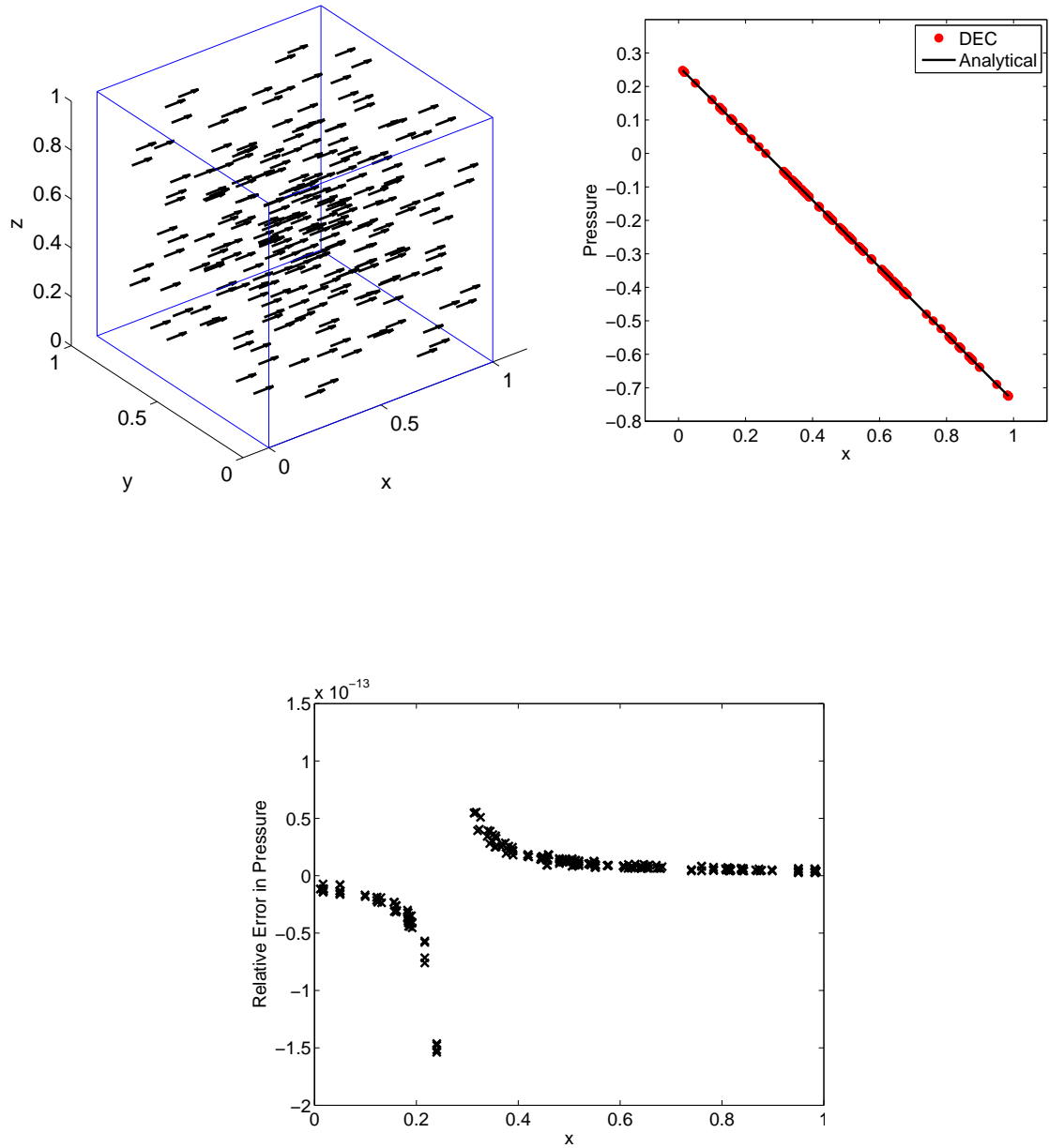

Figure 11: Patch test on a cube. The mesh used for this cube has 244 tetrahedra, each of which is well-centered. Each triangle in the mesh is also well-centered. For clarity, the tetrahedra in the cube have not been shown. The fluid velocity on the boundary is from negative $x$ to positive $x$ direction, with no $y$ or $z$ components. The fluxes (2-cochains) across the internal faces are computed using our method and interpolated using the Whitney map. This is then sampled at the circumcenters, converted into a vector field and plotted as arrows. In the relative error plot for pressure, the data with 0 exact pressure has been removed. 